\title{
Critical Conditions for Connected-k-Coverage in Sensor Networks
}

\author{
Guanqun Yang and Daji Qiao
}

\begin{abstract}
Connected- $k$-coverage has been recognized as an effective notion for prolonging the sensor network lifetime. It requires the monitored region to be $k$-covered by a connected component of active sensors, which is less demanding than requiring $k$-coverage and connectivity among all active sensors simultaneously. While critical conditions for $k$-coverage and connectivity have been studied separately before, there is a lack of fundamental understanding about connected- $k$-coverage. In this paper, as one of the first efforts in understanding the fundamental basics about connected- $k$-coverage, we analyze the critical conditions for connected- $k$-coverage using the percolation theorem and demonstrate their effectiveness using simulation results.
\end{abstract}

Index Terms-Sensor networks, connected-k-coverage, critical conditions, percolation theorem.

\section{INTRODUCTION}

C OVERAGE is one of the fundamental issues to almost all sensor network applications. The ultimate goal of deploying a sensor network is to cover/monitor a region, and in order to have a sensor network function properly, it is always desirable that sensed data can be reported to the user whenever need arises. To achieve this, the requirement of full connectivity among all active sensors is apparently a sufficient condition. However, such a strong requirement may not be necessary. What we really need is the existence of a connected component of active sensors that is able to provide the desired coverage.

Motivated by this observation, we explore the notion of connected-k-coverage as follows. A region is said to be connected- $k$-covered if it is $k$-covered by a connected component of active sensors. Clearly, such a requirement is less demanding than requiring $k$-coverage of the sensing region and connectivity among all active sensors at the same time, but can still guarantee proper functioning of the sensor network. As a result, the number of required active sensors may be reduced, thus prolonging the sensor network lifetime. Unfortunately, there is a lack of fundamental understanding about connected- $k$-coverage such as its critical conditions, which prevents effective and efficient application of the connected$k$-coverage notion to real-world sensor networks.

Critical conditions for $k$-coverage [1], [2] or connectivity [3], [4] alone have been well-studied. Recently, several algorithms [5]-[7] have been proposed to provide connected- $k$ coverage in a sensor network. However, the following fundamental problem remains unstudied: given a randomly deployed sensor network where sensors are active with probability $p$, how many sensors are needed to achieve connected- $k$ coverage? Note that this problem is different from that in [8], where connectivity and $k$-coverage are required at the same time.

Manuscript received May 8, 2008. The associate editor coordinating the review of this letter and approving it for publication was A. Shami.

The authors are with the Department of Electrical and Computer Engineering, Iowa State University, Ames, IA 50011 (e-mail: \{gqyang, daji\}@iastate.edu).

Digital Object Identifier 10.1109/LCOMM.2008.080729.
In this letter, we investigate the theoretical foundations about connected- $k$-coverage and derive its critical conditions under various scenarios. To the best of our knowledge, this is one of the first efforts in understanding the fundamentals about connected- $k$-coverage in sensor networks.

\section{CRITICAL CONDITIONS FOR CONNECTED- $k$-COVERAGE}

\section{A. System Models}

We consider a sensor network with a total of $n$ sensors deployed uniformly randomly and independently to cover a unit-area convex region $(\mathcal{D})$. At any given time, each of the deployed sensors is active with probability $p$, independently of each other. According to [8], [9], for sufficiently large $n$, the set of active sensors form a stationary Poisson point process with density $n p$. Every sensor has fixed sensing radius $\left(r_{s}\right)$ and communication range $\left(r_{c}\right)$, and we let $\alpha=\frac{r_{s}}{r_{c}}$. We denote the sensor network as $N_{\left(r_{s}, r_{c}, n, p\right)}$, and a geometric graph $G_{\left(r_{c}, n, p\right)}$ can be constructed by connecting sensors in $N_{\left(r_{s}, r_{c}, n, p\right)}$ that are within $r_{c}$ to each other. In the rest of this paper, we say that an event almost surely (a.s.) occurs if its probability goes to 1 as $n \rightarrow \infty$. We also write $g(n)=o(f(n))$ iff $\lim _{n \rightarrow \infty} \frac{g(n)}{f(n)}=$ 0 . Throughout this letter, we use $\phi(n p)$ to denote a growing function that goes to infinity as $n \rightarrow \infty$.

\section{B. Critical Condition when $\alpha \leqslant 1$}

LEMMA 1. $\mathcal{D}$ is a.s. $k$-covered by $N_{\left(r_{s}, r_{c}, n, p\right)}$ if, for some growing $\phi(n p), p$ and $r_{s}$ satisfy $n p \pi r_{s}^{2} \geqslant \log (n p)+$ $k \log \log (n p)+\phi(n p)$ for sufficiently large $n$.

Lemma 1 is a well-known critical condition for sensor network $k$-coverage and has been proved in [2]. In [3], the authors derive the sufficient condition for asymptotic connectivity in a randomly uniformly deployed wireless network. When $n p$ is sufficiently large, the underlying point process is essentially a Poisson point process restricted to $\mathcal{D}$ with density $n p$ [9]. Thus, we have the following lemma.

LEMMA 2. $N_{\left(r_{s}, r_{c}, n, p\right)}$ is a.s. connected if, for some growing $\phi(n p), p$ and $r_{c}$ satisfy $n p \pi r_{c}^{2} \geqslant \log (n p)+\phi(n p)$ for sufficiently large $n$.

THEOREM 1. When $\alpha=\frac{r_{s}}{r_{c}} \leqslant 1, \mathcal{D}$ is a.s. connected- $k$ covered by $N_{\left(r_{s}, r_{c}, n, p\right)}$ if, for some growing $\phi(n p), p$ and $r_{s}$ satisfy $n p \pi r_{s}^{2} \geqslant \log (n p)+k \log \log (n p)+\phi(n p)$ for sufficiently large $n$.

Proof: From Lemma 1, we can directly get that $\mathcal{D}$ is a.s. $k$-covered by $N_{\left(r_{s}, r_{c}, n, p\right)}$ under the condition in Theorem 1 . On the other hand, we have

$$
\begin{aligned}
n p \pi r_{c}^{2} & =n p \pi\left(\frac{r_{s}}{\alpha}\right)^{2} \geqslant \frac{\log (n p)+k \log \log (n p)+\phi(n p)}{\alpha^{2}} \\
& >\log (n p)+\phi(n p),
\end{aligned}
$$

for sufficiently large $n$. So, according to Lemma $2, N_{\left(r_{s}, r_{c}, n, p\right)}$ is also a.s. connected under the same condition. Thus, when 
$\alpha \leqslant 1, \mathcal{D}$ is a.s. connected- $k$-covered under the condition in Theorem 1 . Note that, when $\alpha \leqslant 0.5, k$-coverage always implies connectivity [5], [7] and consequently connected- $k$ coverage regardless of $n$.

\section{Critical Condition when $\alpha>1$}

When $\alpha>1$, coverage does not asymptotically imply connectivity. In order to derive the critical condition for this case, we make use of some results from the percolation theorem [10]. According to Proposition 6.6 in [10], as the density of $N_{\left(r_{s}, r_{c}, n, p\right)}$ goes to infinity, there exists a unique dominant connected component consisting of most sensors in $N_{\left(r_{s}, r_{c}, n, p\right)}$, which we denote as $G_{\left(r_{c}, n, p\right)}^{d}$, while each of the sensors not belonging to $G_{\left(r_{c}, n, p\right)}^{d}$ is a.s. isolated (i.e., not connected to any other sensors in $\left.N_{\left(r_{s}, r_{c}, n, p\right)}\right)$. Hence, the probability of $\mathcal{D}$ being connected- $k$-covered (denoted as $P_{\text {ckc }}$ ) is a.s. equal to the probability (denoted as $\hat{P}_{\text {ckc }}$ ) that: (i) $\mathcal{D}$ is $k$-covered by all sensors, and (ii) there does not exist a singly-isolated sensor whose sensing disc is not $k$-covered by sensors belonging to $G_{\left(r_{c}, n, p\right)}^{d}$. We denote the probabilities of satisfying conditions (i) and (ii) as $P_{\text {cov }}$ and $P_{\text {conn }}$, respectively. Then, $\hat{P}_{\mathrm{ckc}}$ is bounded by $\hat{P}_{\mathrm{ckc}} \geqslant P_{\mathrm{cov}}+P_{\mathrm{conn}}-1$. Moreover, if we use $q_{m}$ to denote the probability that a sensor belongs to a connected order- $m$ component of $N_{\left(r_{s}, r_{c}, n, p\right)}$, Proposition 6.6 in [10] can be expressed as $\lim _{n \rightarrow \infty} \frac{1}{q_{1}} \sum_{m=2}^{\infty} q_{m}=0$, i.e., $\sum_{m=2}^{\infty} q_{m}=o\left(q_{1}\right)=o\left(e^{-n p \pi r_{c}^{2}}\right)$. Thus, the probability $\left(P_{a}\right)$ that a sensor belongs to $G_{\left(r_{c}, n, p\right)}^{d}$ can be written as

$P_{a}=1-\sum_{m=1}^{\infty} q_{m}=1-q_{1}-o\left(q_{1}\right)=1-e^{-n p \pi r_{c}^{2}}-o\left(e^{-n p \pi r_{c}^{2}}\right)$.

LEMMA 3. When $\alpha=\frac{r_{s}}{r_{c}}>1, P_{\text {conn }}$ of a sensor network $N_{\left(r_{s}, r_{c}, n, p\right)}$ is a.s. equal to 1 if, for some growing $\phi(n p), p$ and $r_{c}$ satisfy $n p \pi r_{c}^{2}=\frac{\log (n p)+(k+1) \log \log (n p)}{\alpha^{2}}+\phi(n p)$ for sufficiently large $n$.

Proof: Suppose $s$ is an arbitrary sensor in $N_{\left(r_{s}, r_{c}, n, p\right)}$. Let $P_{e}$ denote the conditional probability that, given $s$ is isolated, $s$ ' sensing disc is not $k$-covered by sensors belonging to $G_{\left(r_{c}, n, p\right)}^{d}$. Then, we have

$$
\begin{aligned}
P_{\text {conn }}= & 1-P(\exists \text { a singly-isolated sensor whose sensing disc } \\
& \text { is not } \left.k \text {-covered by sensors belonging to } G_{\left(r_{c}, n, p\right)}^{d}\right) \\
\geqslant & 1-n p \cdot P(s \text { is isolated }) \cdot P_{e}=1-n p \cdot e^{-n p \pi r_{c}^{2}} \cdot P_{e} .
\end{aligned}
$$

We apply Markov's inequality and the crossing technique in [8] to calculate $P_{e}$. Let $N_{k}$ denote the number of crossings within $s$ ' sensing disc (including the disc boundary) that are not $k$-covered by sensors belonging to $G_{\left(r_{c}, n, p\right)}^{d}$. We have

$$
\begin{aligned}
P_{e} \leqslant & P\left(N_{k} \geqslant 2 \mid s \text { is isolated }\right) \leqslant \frac{\mathrm{E}\left(N_{k} \mid s \text { is isolated }\right)}{2} \\
\leqslant & \left(4 n p \pi r_{s}^{2}+2\left(n p \pi r_{s}^{2}\right)^{2}\right)\left(e^{-n p \pi\left(r_{s}^{2}-r_{c}^{2}\right) P_{a}}\right) \\
& \times \sum_{i=0}^{k-1} \frac{\left(n p \pi\left(r_{s}^{2}-r_{c}^{2}\right) P_{a}\right)^{i}}{i !} .
\end{aligned}
$$

Intermediate steps in deriving (4) are omitted due to space limitation; please refer to [11] for details. Now we have

$$
\begin{aligned}
\lim _{n \rightarrow \infty} P_{e} \leqslant & 3\left(n p \pi \alpha^{2} r_{c}^{2}\right)^{2}\left(e^{-n p \pi\left(\alpha^{2}-1\right) r_{c}^{2} P_{a}}\right) \\
& \times k\left(n p \pi\left(\alpha^{2}-1\right) r_{c}^{2} P_{a}\right)^{k-1} .
\end{aligned}
$$

By inserting (5) into (3), we have

$$
\begin{aligned}
\lim _{n \rightarrow \infty} P_{\text {conn }} \geqslant & -C n p\left(e^{-\left(\alpha^{2} P_{a}-P_{a}+1\right) n p \pi r_{c}^{2}}\right) \\
& \times\left(n p \pi r_{c}^{2}\right)^{k+1} P_{a}^{k-1},
\end{aligned}
$$

where $C=3 \alpha^{4} k\left(\alpha^{2}-1\right)^{k-1}$ is a positive constant. To simplify the presentation, we use $p_{1}$ to denote the second term in the right-hand side of (6). By using (2) and the expression of $n p \pi r_{c}^{2}$ in the statement of Lemma 3, we have

$$
\begin{aligned}
& \log p_{1} \\
= & \log (C)+\log (n p)-\alpha^{2} n p \pi r_{c}^{2}+\left(\alpha^{2}-1\right) n p \pi r_{c}^{2} e^{-n p \pi r_{c}^{2}} \\
+ & \left(\alpha^{2}-1\right) n p \pi r_{c}^{2} o\left(e^{-n p \pi r_{c}^{2}}\right)+(k+1) \log \left(n p \pi r_{c}^{2}\right) \\
+ & (k-1) \log \left(1-e^{-n p \pi r_{c}^{2}}-o\left(e^{-n p \pi r_{c}^{2}}\right)\right) \\
= & \log (C)-(k+1) \\
& \log \left(\frac{\alpha^{2} \log (n p) e^{\frac{\alpha^{2} \phi(n p)}{k+1}}}{\log (n p)+(k+1) \log \log (n p)+\alpha^{2} \phi(n p)}\right) \\
+ & \left(\alpha^{2}-1\right) n p \pi r_{c}^{2} e^{-n p \pi r_{c}^{2}}+\left(\alpha^{2}-1\right) n p \pi r_{c}^{2} o\left(e^{-n p \pi r_{c}^{2}}\right) \\
+ & (k-1) \log \left(1-e^{-n p \pi r_{c}^{2}}-o\left(e^{-n p \pi r_{c}^{2}}\right)\right) .
\end{aligned}
$$

As $n \underset{r^{2}}{\rightarrow} \infty, \phi(n p) \quad \rightarrow \quad \infty, \quad$ so $\quad$ we have $e^{-n p \pi r_{c}^{2}} \rightarrow 0, \quad n p \pi r_{c}^{2} e^{-n p \pi r_{c}^{2}} \rightarrow 0$, $\log \left(\frac{\alpha^{2} \log (n p) e^{\frac{\alpha^{2} \phi(n)}{k+1}}}{\log (n p)+(k+1) \log \log (n p)+\alpha^{2} \phi(n p)}\right)^{c} \rightarrow \infty, \quad$ and $n p \pi r_{c}^{2} o\left(e^{-n p \pi r_{c}^{2}}\right) \rightarrow 0$. Accordingly, as $n \rightarrow \infty$, $\log p_{1} \rightarrow-\infty$, i.e., $p_{1} \rightarrow 0$. Hence, $P_{\text {conn }}$ is a.s. equal to 1 .

THEOREM 2. When $\alpha=\frac{r_{s}}{r_{c}}>1, \mathcal{D}$ is a.s. connected$k$-covered by $N_{\left(r_{s}, r_{c}, n, p\right)}$ if, for some growing $\phi(n p), p$ and $r_{c}$ satisfy $n p \pi r_{c}^{2} \geqslant \frac{\log (n p)+(k+1) \log \log (n p)}{\alpha^{2}}+\phi(n p)$ for sufficiently large $n$.

Proof: Firstly, according to the percolation theorem, we know that $P_{\text {ckc }}$ is a.s. equal to $\hat{P}_{\text {ckc }}$, which can be bounded by $\hat{P}_{\text {ckc }} \geqslant P_{\text {cov }}+P_{\text {conn }}-1$. Secondly, the condition for $k$-coverage (as in Lemma 1) can be derived directly from the condition in Theorem 2, i.e., $P_{\text {cov }} \rightarrow 1$ as $n \rightarrow \infty$. Thirdly, according to Lemma 3 , given the condition in Theorem $2, P_{\text {conn }} \rightarrow 1$ as $n \rightarrow \infty$. Therefore, as $n \rightarrow \infty, \hat{P}_{\text {ckc }} \rightarrow 1$, and consequently $P_{\mathrm{ckc}} \rightarrow 1$

\section{Summary}

We summarize the results of this work as follows.

- When $\alpha \leqslant 1$ (i.e., $r_{s} \leqslant r_{c}$ ), $\mathcal{D}$ is a.s. connected- $k$ covered under the condition in Theorem 1. Particularly, when $\alpha \leqslant 0.5, k$-coverage always implies connectivity and consequently connected- $k$-coverage regardless of $n$.

- When $\alpha>1$ (i.e., $r_{s}>r_{c}$ ), the derived critical condition for connected- $k$-coverage a.s. implies $k$-coverage, but the network is a.s. disconnected. Compared with the critical condition for connectivity in Lemma 2, the derived criti- 


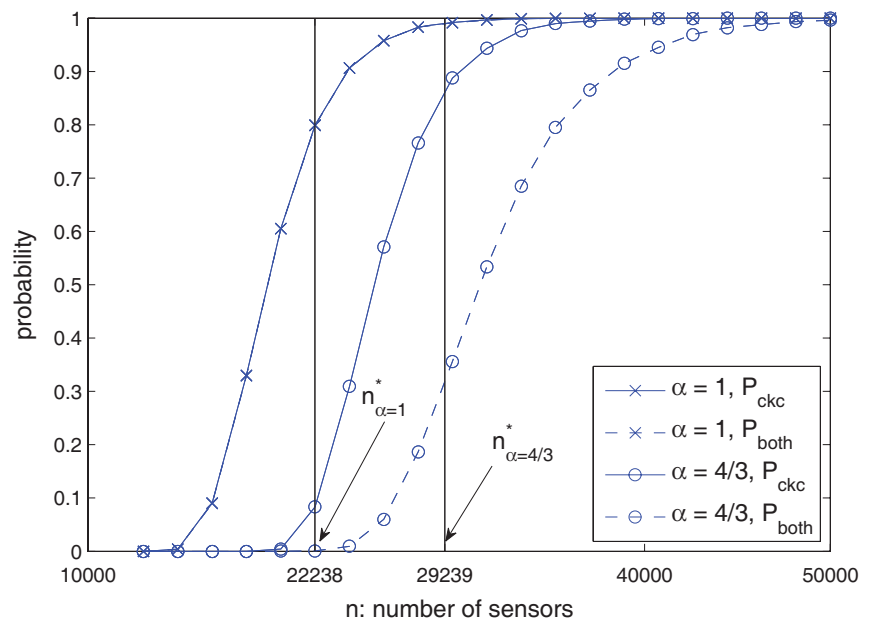

Fig. 1. $\quad P_{\text {ckc }}$ and $P_{\text {both }}$ for different combinations of $n$ and $\alpha . n$ is the total number of deployed sensors. At any given time, each sensor is active with probability $p=0.1$. $\alpha$ is the ratio of the sensing radius $\left(r_{s}\right)$ to the communication range $\left(r_{c}\right), n_{\alpha}^{*}$ 's are obtained using (7).

cal condition for connected- $k$-coverage is less demanding than that for simultaneous $k$-coverage and connectivity.

\section{Simulation RESUlts}

\section{A. Simulation Setup}

Due to space limitation, we only show the simulation results for connected-1-coverage. We consider a unit-area square region to be monitored by a network of $n$ sensors. At any give time, each sensor is active with probability $p=0.1$, independently of each other. For each sensor, $r_{s}$ is 0.04 units, and $r_{c}$ varies from 0.03 to 0.04 units, which correspond to different $\alpha$. We choose the slow growing $\sqrt{\log \log (n p)}$ as $\phi(n p)$. We divide the monitored region into a grid of size $250 \times 250$, and approximate that connected-1-coverage is achieved if all grid points are 1-covered by sensors on $G_{\left(r_{c}, n, p\right)}$. The torus convention [8] is employed in the simulation to avoid edge effects.

\section{B. Simulation Results}

For each combination of $n$ and $\alpha, 10000$ sensor network deployments are randomly generated. We simulate one snapshot for each deployment, where each sensor is active with probability $p=0.1$. Thus, the probability of connected-1coverage can be estimated as $P_{\mathrm{ckc}}=\frac{M}{10000}$, where $M$ is the total number of snapshots when all points are 1-covered by sensors on $G_{\left(r_{c}, n, p\right)}$. We also study $P_{\text {both }}$ - the probability that both 1-coverage of the region and connectivity of active sensors are satisfied. Simulation results are plotted in Fig. 1 and we have the following observations.

Firstly, when $\alpha=1$, the difference between two probability curves is too small to be visible for any $n$. Similar results can be observed for other $\alpha \leqslant 1$ cases. This implies that, when $\alpha \leqslant 1$, though we only prove (in Theorem 1) the asymptotic implication of 1-coverage on connectivity, requiring connected-1-coverage is indeed almost always equivalent to the requirement that both 1-coverage of the region and connectivity among active sensors are satisfied, regardless of the size of the sensor network.
Secondly, when $\alpha=4 / 3$, there is a significant gap between the two probability curves. In fact, the gap gets larger as $\alpha$ increases. As shown in the figure, when $P_{\mathrm{ckc}}$ is very close to $1, P_{\text {both }}$ is still low. This implies that, though a network of active sensors is still disconnected, it may have already been able to connected-1-cover the region.

At last, we calculate $n_{\alpha}^{*}$ as follows and show it in the figure:

$$
n_{\alpha}^{*}=\left\{\begin{array}{rlrl}
\min \left\{n: n p \pi r_{s}^{2} \geqslant\right. & \log (n p)+k \log \log (n p) & \\
& +\phi(n p)\}, & & \text { if } \alpha \leqslant 1, \\
\min \left\{n: n p \pi r_{c}^{2} \geqslant\right. & \frac{\log (n p)+(k+1) \log \log (n p)}{\alpha^{2}} & & +\phi(n p)\},
\end{array}\right.
$$

By Theorems 1 and 2, we expect that when $n \geqslant n_{\alpha}^{*}, P_{\text {ckc }}$ should be close to 1 . However, we see from the figure that this prediction is not so accurate. The discrepancies are due to the asymptotic nature of our results, and we expect them to become smaller as $n$ grows or when a faster growing function is chosen for $\phi(n p)$, e.g., $(\log \log (n p))^{\frac{2}{3}}$. This observation also indicates that in practice a slightly higher value of $n$ should be used than the one predicted by $n_{\alpha}^{*}$ in (7).

\section{CONCLUSIONS}

In this paper, we explore the notion of connected- $k$ coverage in sensor networks. We investigate the theoretical foundations about connected- $k$-coverage and, by applying the percolation theorem, we derive the critical conditions for connected- $k$-coverage under different relations between sensors' sensing radius and communication range. Simulation results show how the derived critical conditions may be used in practice for predicting the number of active sensors required to achieve the desired connected- $k$-coverage, given the region to be covered and sensors' sensing radius and communication range.

\section{ACKNOWLEDGMENTS}

The research reported in this paper was supported in part by the Information Infrastructure Institute (iCube) of Iowa State University and the NSF under Grant CNS 0716744.

\section{REFERENCES}

[1] H. Zhang and J. C. Hou, "Is deterministic deployment worse than random deployment for wireless sensor networks?" in Proc. IEEE Infocom, 2006.

[2] S. Kumar, T. H. Lai, and J. Balogh, "On k-coverage in a mostly sleeping sensor network," in Proc. ACM MobiCom, Sept. 2004.

[3] P. Gupta and P. Kumar, "Critical power for asymptotic connectivity," in Proc. 37th IEEE Conference on Decision and Control, Dec. 1998.

[4] H. Zhang and J. C. Hou, "On the critical total power for asymptotic kconnectivity in wireless networks," in Proc. IEEE Infocom, Mar. 2005.

[5] — - "Maintaining sensing coverage and connectivity in large sensor networks," Wireless Ad Hoc and Sensor Networks: An International Journal, vol. 1, no. 1, pp. 89-124, 2005.

[6] Z. Zhou, S. Das, and H. Gupta, "Variable radii connected sensor cover in sensor networks," in Proc. IEEE SECON, Oct. 2004.

[7] X. Wang, G. Xing, Y. Zhang, C. Lu, R. Pless, and C. Gill, "Integrated coverage and connectivity configuration in wireless sensor networks," ACM Trans. Sensor Networks, vol. 1, no. 1, pp. 36-72, 2005.

[8] W. Mo, D. Qiao, and Z. Wang, "Lifetime maximization of sensor networks under connectivity and k-coverage constraints," in Proc. IEEE DCOSS, June 2006.

[9] P. Hall, Introduction to the Theory of Coverage Process. John Wiley \& Sons, 1988.

[10] R. Meester and R. Roy, Continuum Percolation. Cambridge University Press, 1996.

[11] Tech. Rep., http://www.public.iastate.edu/ gqyang/clrpt.pdf. 